\title{
Normas de Orientação aos Pacientes para Armazenagem e Conservação de Imunobiológicos
}

\author{
Patients Guidelines for Immunobiologicals Storage and Conservation
}

\author{
Tássia Moraes de Assis Damasceno*a; Vander Fernandes ${ }^{\mathrm{a}}$; Boris Felsky dos Anjos ${ }^{\mathrm{a}}$; Thaissa Araujo Rachid Jaudy; \\ Aline Bruehmueller Ale ${ }^{\text {a }}$ Marcelo Neves Lotufo ${ }^{a}$; Henrique César Santejo Silveira ${ }^{a}$
}

aUniversidade de Cuiabá, Programa de Pós-Graduação Stricto Sensu em Ambiente e Saúde. MT, Brasil.

*E-mail: dra.tassia.damasceno@gmail.com

\begin{abstract}
Resumo
As doenças reumáticas autoimunes, tais como: artrite reumatoide, espondilite anquilosante, artrite psoriásica são doenças inflamatórias crônicas, que provocam deformidades permanentes. O tratamento dessas patologias envolve o uso de medicamentos imunobiológicos, que se caracterizam por ser termolábeis e de uso parenteral, necessitando de cuidados com a armazenagem em temperatura recomendada para a manutenção de sua eficácia e segurança. Estudos observacionais vêm demonstrando que a maioria dos pacientes armazena seus medicamentos imunobiológicos fora da temperatura recomendada em bula, e houve associação entre temperatura incorreta do armazenamento destas drogas e moderada/alta atividade das doenças reumáticas autoimunes. Orientar aos pacientes os cuidados de transporte, de armazenagem e de conservação dos medicamentos imunobiológicos em domicílio, visando preservar a eficácia e segurança do tratamento. Foram pesquisadas publicações sobre o armazenamento domiciliar dos medicamentos biológicos nas bases de dados virtuais e manuais de conservação dos orgãos de regulamemtação de armazenagem de biomedicamentos, relacionando terapia biológica, armazenamento doméstico e doenças reumáticas. Com a revisão de literatura foi possível elaborar normas quanto ao cuidado no transporte, no armazenamento e na conservação dos medicamentos imunobiológicos, respeitando a realidade local. Com o acesso às normas de armazenamento e conservação dos medicamentos imunobiológicos, os pacientes obtiveram maior conhecimento e, assim, uma resposta mais eficaz, diminuindo também a chance de eventos adversos durante o seu tratamento.
\end{abstract}

Palavras-chave: Terapia Biológica. Temperatura Ambiental. Doenças Reumáticas. Antirreumáticos. Armazenamento Doméstico.

\begin{abstract}
Autoimmune rheumatic diseases such as rheumatoid arthritis, ankylosing spondylitis, psoriatic arthritis are chronic inflammatory diseases that cause permanent deformities. The treatment of these pathologies involves the use of immunobiological drugs that are characterized by being thermolabile and for parenteral use, requiring care with storage at the recommended temperature to maintain their effectiveness and safety. Observational studies have shown that most patients store their immunobiological drugs outside the recommended temperature in the medicine package insert, and there was an association between incorrect storage temperature of such drugs and moderate / high autoimmune rheumatic diseases activity. Objective: To guide patients in the care of transport, storage and preservation of immunobiological drugs at home in order to preserve the treatment effectiveness and safety. Methods: Publications on the home storage of biological medicines were searched in the virtual databases and manuals for the conservation of biomedical storage regulatory bodies, concerning biological therapy and home storage. Result: With the literature review, it was possible to elaborate rules regarding the care in the transport, storage and conservation of immunobiological drugs, respecting the local reality. Conclusions: With access to the rules for storage and conservation of immunobiological drugs, patients obtained greater knowledge and thus a more effective response, also decreasing the chance of adverse events during their treatment.
\end{abstract}

Keywords: Biological Therapy. Temperature. Rheumatic Diseases. Antirheumatic Agents. Home Storage.

\section{Introdução}

Doenças autoimunes envolvem um grupo de doenças, em que o sistema imunológico é ativado por fatores diversos em pacientes geneticamente susceptíveis, provocando uma resposta orgânica destrutiva contra o próprio tecido ou órgão do corpo humano (RITZ et al., 2010). Corresponde uma série de doenças sistêmicas, tais como: artrite reumatoide, psoríase, espondilite anquilosante, entre outras, cujo aparecimento ou sua exacerbação podem ser influenciados por infecções, imunodeficiência primária, fatores imunológicos adjuvantes e outros fatores ambientais (FARHAT et al., 2011). O tratamento das doenças reumáticas autoimunes sofreu uma progressiva melhora, ao longo da última metade do século passado, que foi expandida com a contribuição das terapias biológicas ou imunobiológicos (também denominados agentes biológicos ou medicamentos modificadores do curso da doença biológicos - MMCD-b) (MOTA et al., 2015; TAK et al., 2011; UPCHURCH et al., 2012).

Estas medicações são termolábeis e de uso parenteral, logo, sua correta armazenagem e administração estão diretamente relacionadas à eficácia e segurança. Estudos demostraram que a maioria dos pacientes não armazena os medicamentos imunobiológicos dentro dessa faixa de temperatura recomendada em nível domiciliar (CUELLAR et al., 2010; DAMASCENO et al. 2020; VLIELAND et al., 2016). A consequência mais comum de expor proteínas a condições 
de temperatura inadequadas é a formação de agregados (LIU et al., 2014; ROSENBERG et al., 2006; VLIELAND et al., 2018), que podem levar ao desenvolvimento de anticorpos antidrogas e à diminuição da eficácia do medicamento, bem como ao aumento da probabilidade de efeitos colaterais (GARCES; DEMENGEOT, 2012).

O objetivo do trabalho foi orientar os pacientes quanto aos cuidados no transporte, armazenagem e conservação dos medicamentos imunobiológicos em domicílio, visando preservar a eficácia e segurança do tratamento, de acordo com as normas de conservação em bula e manuais da Agência Nacional de Vigilância Sanitária. A população alvo a ser instruída são os pacientes adultos assistidos no ambulatório de reumatologia do Hospital Geral em Cuiabá-MT, com diagnóstico de artrite reumatoide, espondilite anquilosante e artrite psoriásica em uso de imunobiológicos injetáveis subcutâneos - Adalimumabe, Etanercepte, Golimumabe, Secuquinumabe, Certolizumabe pegol, Abatacepte, sendo estes armazenados no domicílio.

\section{Desenvolvimento}

\subsection{Metodologia}

Foi realizado um levantamento nas bases de dados virtuais correlacionando doenças reumáticas e terapia biológica e armazenamento domiciliar. Também uma pesquisa nos manuais de conservação dos biomedicamentos do Ministério da Saúde - Brasil. E coleta das informações contidas nas bulas dos medicamentos imunobiológicos injetáveis parenterais (Adalimumabe, Etanercepte, Golimumabe, Secuquinumabe, Certolizumabe pegol, Abatacepte).

\subsection{Resultados}

As medicações imunobiológicas utilizadas no tratamento das doenças reumáticas autoimunes consistem em moléculas semelhantes às humanas, como imunoglobulinas, que bloqueiam mediadores inflamatórios específicos envolvidos na fisiopatologia destas doenças (MOTA et al., 2015). Entre as doenças reumáticas autoimunes, nas quais estas drogas são utilizadas se destacam: a artrite reumatoide, artrite psoriásica e espondilite anquilosante (MOTA et al., 2012; SAMPAIO-BARROS et al., 2007; CARNEIRO et al., 2013; SAMPAIO-BARROS et al., 2013). De acordo com a Agência Nacional de Vigilância Sanitária - ANVISA, estas medicações estão contempladas no Sistema Único de Saúde - SUS e o acesso pelos pacientes se faz pelas Secretarias Estaduais de Saúde através do componente especializado da assistência farmacêutica, que dispensam estas medicações diretamente aos pacientes, ficando a cargo destes a armazenagem e administração (BRASIL, 2010, 2012a, 2018a, 2018b). Essas medicações devem ser mantidas em sua embalagem original e armazenadas entre 2 e $8{ }^{\circ} \mathrm{C}$ (BRASIL, 2012b). Realizou-se um estudo epidemiológico, descritivo, do tipo transversal, em que foram avaliados e triados os pacientes do ambulatório de reumatologia no Hospital Geral de Cuiabá - MT, em 2017/2018 (DAMASCENO et al., 2020). Foram incluídos: pacientes com diagnóstico de artrite reumatoide, artrite psoriásica e espondilite anquilosante em uso de terapias biológicas de uso injetável subcutâneo, adultos de ambos os sexos, residentes em Cuiabá e Várzea Grande-MT, que armazenavam o medicamento imunobiológico no domicílio. Foi realizada uma visita domiciliar e aferida a temperatura de armazenamento do medicamento imunobiológico no domicílio, utilizando um termômetro digital adequado para essa aferição.

Este estudo demonstrou que, na população estudada, a maioria dos pacientes $(65 \%)$ com doenças reumáticas autoimunes não armazena seus medicamentos imunobiológicos dentro da faixa de temperatura recomendada pelo fabricante. Este resultado está de acordo com publicações anteriores sobre condições de armazenamento doméstico para medicamentos imunobiológicos, como descrito por Vlieand et al. (2016) e Cuellar et al. (2010). Nesta pesquisa se verificou também que o armazenamento em temperatura incorreta apresentou associação com a atividade moderada/alta da doença reumática, sendo $76 \%$ maior a ocorrência de armazenamento em temperatura inadequada entre os pacientes em moderada/ alta atividade da doença reumática. Em relação à segurança no tratamento com MMCD-b não foram encontradas diferenças estatisticamente significativas em relação à associação de reações adversas e armazenamento em temperatura correta ou incorreta do MMCD-b. A partir da conclusão deste estudo feito nesta região, e também de outras publicações relatando o mesmo desfecho ao redor do Mundo se verificou a necessidade de criar uma normativa para os pacientes assistidos no ambulatório de reumatologia do Hospital Geral em Cuiabá MT sobre os cuidados com o armazenamento domiciliar dos medicamentos imunobiológicos.

Normas sobre o armazenamento e conservação dos medicamentos imunobiológicos injetáveis subcutâneos:

1. o medicamento imunobiológico deve ser retirado e armazenado no isopor ou bolsa térmica com gelo;

2. deve ser mantido na embalagem original, não retirar da caixa;

3. não agitar ou sacudir o medicamento, evite impactos durante a retirada na farmácia de alto custo até o seu domicílio;

4. no domicílio, o medicamento imunobiológico deve ser armazenado na geladeira em sua embalagem original;

5. na geladeira, o medicamento imunobiológico deve ser colocado na prateleira;

6. não revestir a embalagem do medicamento imunobiológico com saco plástico;

7. evitar ficar abrindo a geladeira muitas vezes, por conta da perda da cadeia de frio;

8. manter a temperatura da sua geladeira entre 2 e $8^{\circ} \mathrm{C}$;

9. não deixe o medicamento imunobiológico em temperatura ambiente por muito tempo, retire da geladeira somente no momento da aplicação.

10. em caso de dúvida sobre o armazenamento e conservação do seu medicamento imunobiológico no domicílio, entre em contato com o seu médico assistente, ou ligue para o Serviço de Terapia Assistida de referência. 


\section{Conclusão}

Com o acesso às normas de armazenamento e conservação dos medicamentos imunobiológicos, os pacientes obtiveram maior conhecimento e, assim, uma resposta mais eficaz, diminuindo também a chance de eventos adversos durante o seu tratamento.

\section{Referências}

BRASIL. Boletim brasileiro de avaliação de tecnologias em saúde: medicamentos biológicos para o tratamento da artrite reumatoide. Brasília: MS, ANVISA, 2012a.

BRASIL. RDC $\mathrm{n}^{\circ}$ 45, de 9 de agosto de 2012: dispõe sobre a realização de estudos de estabilidade de insumos farmacêuticos ativos. Brasília: MS, 2012 b.

BRASIL. Grupo de Trabalho para discussão e formulação da Política Nacional de Medicamentos Biológicos no âmbito do Sistema Único de Saúde. Portaria $\mathrm{n}^{\mathrm{o}} 1.160$, de 3 maio de 2018a. Brasília: MS, 2018a.

BRASIL. Relação Nacional de Medicamentos Essenciais. Brasília-DF: Ministério da Saúde, Secretaria de Ciência, Tecnologia e Insumos Estratégicos, Departamento de Assistência Farmacêutica e Insumos Estratégicos. Brasília: MS, 2018b.

BRASIL. RDC 55, de 16 de dezembro 2010. Brasília: MS, 2010.

BULA CIMZIA $^{\circledR}$ (certolizumabe pegol). AstraZeneca do Brasil Ltda. Disponível em: <http://www.anvisa.gov.br/datavisa/fila bula>. Acessado em: 14 jan. 2020.

BULA COSENTYX ${ }^{\circledR}$ (secuquinumabe). Novartis Biociências S.A. Disponível em: <https://portal.novartis.com.br>. Acesso em: 14 jan. 2020.

BULA ENBREL ${ }^{\circledR}$ (etanercepte). Disponível em: <http://www. pfizer.com.br/produtos. aspx? Acessibilidade $=$ false $>$. Acesso em: 14 jan. 2020.

BULA HUMIRA $^{\circledR}$ (adalimumabe). Abbott Laboratórios do Brasil Ltda. Disponível em: <http://www4.anvisa.gov.br/base/visadoc/ BM/BM[34626-1-0].PDF>. Acesso em 14 dez 2020.

BULA ORENCIA ${ }^{\circledR}$ (abatacepte). Disponível em: $<$ http://www. bristol.com.br/produtos. aspx>. Acesso em: $14 \mathrm{dez} 2020$.

BULA SIMPONI ${ }^{\circledR}$ (golimumabe). Janssen-Cilag Farmacêutica Ltda. Disponível em: <http://www.anvisa.gov.br/datavisa/fila bula>. Acesso em: 14 dez. 2020.

CARNEIRO, S. et al. Recomendações sobre diagnóstico e tratamento da artrite psoriásica. Rev Bras Reumatol., v.53, n.3, v.227-241, 2013.

CUELLAR, M.J. et al. Quality of storage of thermolabile drugs in patients' homes. Rev Calid Asist., n. 25, p.64-69, 2010. doi: 10.1016/j.cali.2009.09.001.
DAMASCENO, T.M.A. et al. Storage conditions of immunobiologicals and their influence on the efficacy and safety in the treatment of autoimmune rheumatic diseases. Open Rheumatol. J., v. 14, p. 1-6, 2020. doi: 10.2174/1874312902014010001.

FARHAT, S.C.L. et al. Air pollution in autoimmune rheumatic diseases: a review. Autoimmunity Rev., v. 11, p. 14-21, 2011. doi: 10.1016/j.autrev.2011.06.008.

GARCES, S.; DEMENGEOT, J. The immunogenicity of anti-TNF therapy in immune-mediated inflammatory diseases: a systematic review of the literature with a meta-analysis. Ann Rheum Dis., v. 72, p. 1947-1955, 2012. doi: 10.1136/annrheumdis-2012-202220.

LIU, L. et al. Freezing-induced perturbation of tertiary structure of a monoclonal antibody. J Pharm Sci., v. 103, n. 7, p.19791986, 2014. doi: 10.1002/jps.24013.

MOTA, E. et al. Consenso 2012 da Sociedade Brasileira de Reumatologia para o tratamento da artrite reumatoide. Rev. Bras. Reumatol., v.52, n.2, p.135-174, 2012.

MOTA, E. et al. Segurança do uso de terapias biológicas para o tratamento de artrite reumatoide e espondiloartrites. Rev. Bras. Reumatol., v. 55, n. 3, p. 281-309, 2015.

RITZ, S.A. et al. Air pollution as a potential contributor to the 'epidemic' of autoimmune disease. Med. Hypotheses, v. 74, p. 110-117, 2010. doi: 10.1016/j.mehy.2009.07.033.

ROSENBERG, A.S. et al. Effects of protein aggregates: an immunologic perspective. AAPS J., v. 8, p. 501-507, 2006. doi: $10.1208 /$ aapsj080359.

SAMPAIO-BARROS, P. et al. Consenso Brasileiro de Espondiloartropatias: espondilite anquilosante e artrite psoriásica - diagnóstico e tratamento - primeira revisão. Rev. Bras. Reumatol., v. 47, n. 4, p. 233-242, 2007.

SAMPAIO-BARROS, P. et al. Recomendações sobre diagnóstico e tratamento da espondilite anquilosante. Rev. Bras. Reumatol., v. 53, n. 3, p. 242-257, 2013.

TAK, P.P. et al. Advances in rheumatology: new targeted therapeutics. Arthritis Res Ther., v.13, p.3, 2011. doi: 10.1186/1478-6354-13-S1-S5.

UPCHURCH, K.S. et al. Evolution of treatment for rheumatoid arthritis. Rheumatology (Oxford), v.5, n.6, p.28-36, 2012. doi: 10.1093/rheumatology/kes278.

VLIELAND, N.D. et al. The impact of inadequate temperature storage conditions on aggregate and particle formation in drugs containing tumor necrosis factor-alpha inhibitors. Pharm Res., v. 35, n. 2, p. 42, 2018. doi: 10.1007/s11095-017-2341-x.

VLIELAND, N.D. et al. The majority of patients do not store their biologic disease-modifying antirheumatic drugs within the recommended temperature range. Rheumatology, v.55, p. 704709, 2016. doi: 10.1093/rheumatology/kev394. 